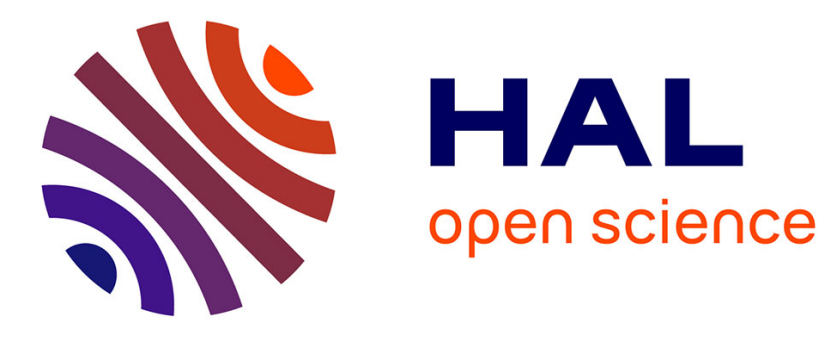

\title{
Suivi du transport des marchandises en ville: un nouveau pacte entre enquêtes statistiques et modélisation
}

Florence Toilier, Alain Bonnafous, Jean-Louis Routhier

\section{- To cite this version:}

Florence Toilier, Alain Bonnafous, Jean-Louis Routhier. Suivi du transport des marchandises en ville: un nouveau pacte entre enquêtes statistiques et modélisation. Annales des mines - Série Responsabilité et environnement, 2014, Transports terrestres et développement économique, 75, pp.57-64. halshs01855542

\section{HAL Id: halshs-01855542 \\ https://shs.hal.science/halshs-01855542}

Submitted on 27 Aug 2018

HAL is a multi-disciplinary open access archive for the deposit and dissemination of scientific research documents, whether they are published or not. The documents may come from teaching and research institutions in France or abroad, or from public or private research centers.
L'archive ouverte pluridisciplinaire HAL, est destinée au dépôt et à la diffusion de documents scientifiques de niveau recherche, publiés ou non, émanant des établissements d'enseignement et de recherche français ou étrangers, des laboratoires publics ou privés. 


\section{Suivi du transport des marchandises en ville : un nouveau pacte entre enquêtes statistiques et modélisation}

Par Florence TOILIER, Alain BONNAFOUS et Jean-Louis ROUTHIER *

Les transports de marchandises en ville ont été longtemps mal connus par comparaison à ce que l'on sait des transports urbains de personnes, y compris en matière de simulations de déplacements réalisées sous différentes hypothèses de politique de transport. On soupçonne pourtant depuis longtemps que ces transports dits utilitaires puissent avoir des effets sur la congestion de la voirie du même ordre que ceux que peut avoir la voiture particulière. C'est à l'initiative du ministère de l'Équipement qu'un programme national de recherche a été lancé il y a vingt ans de cela pour améliorer nos statistiques et, plus généralement, nos connaissances sur cette logistique urbaine. Cet article présente la mise en œuvre d'enquêtes statistiques inédites qui ont été conçues en accointance avec le modèle qu'elles ont permis de valider et donne quelques illustrations de l'avancement des connaissances sur le fret urbain.

Les politiques urbaines en matière de transport s'appuient sur des instruments de prévision et de simulation des trafics de voyageurs dont on peut dire qu'ils ont atteint une certaine maturité. C'est ainsi que, dans le domaine de la modélisation des déplacements des personnes, on peut observer qu'il existe aujourd'hui une palette de modèles opérationnels qui reposent sur un standard de connaissances et de formalisation établi depuis quelques décennies. Ce standard est concrétisé, par exemple, par le fait que quatre modèles se partagent l'essentiel du marché européen des modèles de trafic (Visem/Visum (1), Cube (2), TransCAD (3) et Emme3 (4)). À ces modèles correspondent bien entendu des statistiques dont l'essentiel résulte d'enquêtes-ménages sur les déplacements, qui, elles-mêmes, reposent sur une méthodologie standardisée depuis les années 1970.

Mais nous sommes loin du compte en matière de fret urbain. Nous nous proposons pourtant de montrer comment ce retard dans nos connaissances sur les transports de marchandises en ville a pu être partiellement comblé au cours des dernières années, en particulier sur la base de travaux réalisés en France.

Partant du constat de nos ignorances (en dépit de financements réguliers de recherches sur le sujet), le gouvernement français a souhaité dans les années 1990 mettre en œuvre un vaste programme national de recherche nommé "Marchandises en ville ". Ce program- me prévoyait en particulier le financement d'une enquête lourde, dont la conception a été confiée au LET (5).

Le travail de réflexion méthodologique préalable a reposé sur une constatation simple : la relative efficacité des enquêtes sur la mobilité des personnes qui alimentent les modèles de déplacements urbains tient au fait que ces enquêtes ont été progressivement conçues en fonction de la spécification de ces modèles. II y a eu ainsi une forte corrélation entre la pertinence de moins en moins discutable des modèles successifs et la pertinence des informations statistiques. Un pacte historique en quelque sorte a été passé entre les enquêtes et les modèles, en ce sens que le succès des unes et des autres se sont mutuellement entretenus.

Voyons tout d'abord le modèle.

La réflexion qui était alors commanditée sur la conception d'une enquête inédite a consisté à rechercher un pacte comparable. Pour cela, il était nécessaire d'esquisser une modélisation des transports de marchandises en ville qui n'existait pas encore. Nous savions seulement que cette modélisation ne pouvait être une simple transposition à un milieu urbain des modèles de fret interrégionaux, car toutes les tentatives en ce sens avaient jusqu'alors échoué : toutes s'étaient heurtées à cette évidence qu'un modèle qui simule des tonnes ou des tonnes-kilomètres n'avait guère de sens dans un espace où une tonne de fret peut parfois être transportée en un seul lot (s'il s'agit d'une 
palette d'eau minérale, par exemple), mais aussi parfois en quelques milliers de lots (s'il s'agit des médicaments livrés quotidiennement à des pharmacies, par exemple).

L'objectif d'un éventuel modèle n'étant donc pas, à l'évidence, de formaliser puis de simuler des flux de marchandises mesurés en tonnes ou en tonnes-kilomètres, quel peut-il bien être ? Cet objectif est tout simplement de traiter du problème économique qui est par définition un problème de rareté, en l'occurrence la rareté de l'espace de voirie, qu'aggravent les activités de livraison.

D'où un objectif simple pour une enquête et pour le modèle que celle-ci doit alimenter : analyser et formaliser l'occupation de la voirie par l'activité du transport de marchandises en ville en partant de l'intuition que cette occupation résulte tout autant du stationnement sur la voirie lors d'une livraison (ou lors d'un enlèvement) que du déplacement du véhicule. Cette hypothèse intuitive, qui allait être largement vérifiée, conduit tout naturellement à retenir le mouvement, c'est-à-dire la livraison ou l'enlèvement, comme unité d'observation, dans la modélisation comme dans l'enquête qui lui est nécessaire.

Cette option méthodologique correspond à une rupture avec le paradigme dominant des transports de personnes qui repose sur le remplissage de matrices origine-destination par des nombres de déplacements. Pour bien comprendre cette rupture, il suffit de comparer l'unité d'observation "déplacements " (transport de personnes) à l'unité d'observation "mouvements " (transport de marchandises), que résume le Tableau ci-dessous.

II s'agissait donc de poser les bases d'un modèle cohérent avec un objectif clair, celui de rendre compte des mouvements et de leurs conséquences sur l'encombrement de la voirie. On voit ainsi apparaitre les principaux éléments du cahier des charges de cette modélisation. Par exemple, la restitution d'un mouvement devra rendre compte des conditions de l'arrêt du véhicule de livraison : horaire, durée, arrêt hors voirie ou sur voirie, taille du véhicule, etc. Le modèle doit aussi permettre de reconstituer les déplacements induits, dont on peut imaginer qu'ils ne correspondent pas à la même occupation de la voirie, selon que le mouvement correspond à une livraison unique ou selon qu'il s'inscrit dans une tournée comportant plusieurs dizaines de livraisons.

C'est dans cet esprit qu'a été conçu le modèle FRETURB, qui est aujourd'hui identifié (Russo \& Coml, 2010) comme ayant initié une nouvelle famille de modèles, les deliveries models (6), et qui a été présenté sous sa forme initiale en 1998 (7). Régulièrement développé depuis, ce modèle permet de restituer et de simuler des trafics urbains liés aux transports de marchandises avec des variables exogènes, dont les valeurs sont généralement disponibles dans le répertoire SIRENE (8) de I'INSEE. Cela signifie que ce modèle peut être utilisé en faisant l'économie du coût considérable d'une enquête statistique ad hoc sur les transports de marchandises : une caractéristique qui explique qu'il soit le seul à être appliqué dans une quarantaine de villes françaises et de pays voisins (représentées sur la carte de la page suivante).

Nous n'entrerons pas ici dans les détails de la spécification de ce modèle (9), mais nous préciserons simplement que sa première hypothèse centrale est que le nombre moyen hebdomadaire de mouvements liés à un établissement est supposé être déterminé par l'activité dudit établissement et par sa taille. La seconde hypothèse centrale est que les mouvements d'une même catégorie d'établissement sont répartis, selon des proportions stables, dans des catégories logistiques bien identifiées par type de véhicule et par type de tournée. Les premiers résultats des enquêtes statistiques lourdes ont permis de vérifier ces hypothèses.

\section{Trois enquêtes inédites}

Entre 1994 et 1997, trois enquêtes ont en effet été réalisées dans des villes de tailles différentes (Marseille, Bordeaux et Dijon), sous le contrôle méthodologique du LET, qui en a également assuré le traitement statistique et l'analyse des résultats (10).

Le mouvement étant retenu comme unité d'observation, il convenait de délimiter le champ d'étude et, en particulier, de préciser l'objet des enquêtes dans ce système complexe que constitue la logistique urbaine. Cette

\begin{tabular}{|c|c|c|}
\hline & Transports de personnes & Transports de marchandises \\
\hline Unité d'observation : & Un déplacement & Un mouvement (livraison ou enlèvement) \\
\hline Définie par: & $\begin{array}{l}\checkmark \text { Un lieu et un motif au départ. } \\
\checkmark \text { Un lieu et un motif à l'arrivée. }\end{array}$ & $\begin{array}{l}\checkmark \text { L'activité, la nature, la taille et la locali- } \\
\text { sation de l'établissement concerné. } \\
\checkmark \text { Informations sur la tournée (nombre } \\
\text { d'arrêts, type de véhicule utilisé, opéra- } \\
\text { teur effectuant le transport). }\end{array}$ \\
\hline Et enrichie de : & $\begin{array}{l}\checkmark \text { Heures de départ et d'arrivée. } \\
\checkmark \text { Mode de transport. } \\
\checkmark \text { Caractéristiques de l'individu. }\end{array}$ & $\begin{array}{l}\checkmark \text { Durée et conditions de l'arrêt du véhicu- } \\
\text { le. } \\
\checkmark \text { Fréquence et rythmes. }\end{array}$ \\
\hline
\end{tabular}

Comparaison entre les statistiques de mobilité " voyageurs " et " marchandises ". 


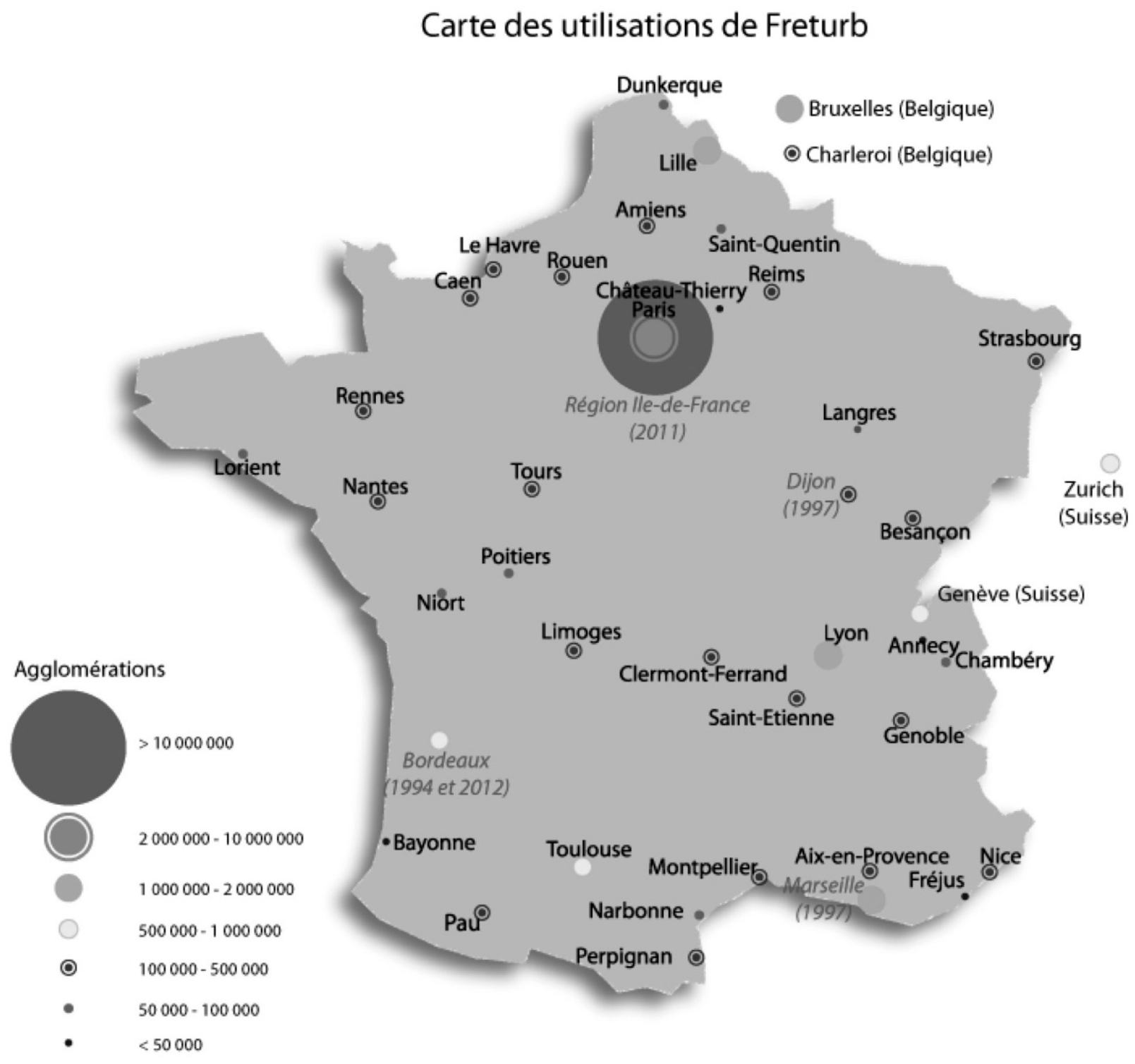

Agglomérations enquêtées

expression, logistique urbaine, est désormais employée pour désigner le sous-système urbain relatif aux déplacements des biens dans la ville. On peut le représenter de manière simplifiée par le schéma de la page suivante (voir le Schéma 1) dans lequel toutes les flèches désignent des flux de colis de natures différentes.

Pour réduire cette complexité, on peut décomposer la logistique urbaine en trois segments :

$\checkmark$ les échanges de marchandises entre établissements, que ces établissements aient une activité industrielle, commerciale ou tertiaire... Ces échanges représentent de 35 à $40 \%$ du total des kilomètres générés par le transport de marchandises en milieu urbain, du lundi au vendredi, soit 5 à $10 \%$ du trafic urbain total en unités de véhicule particulier (UVP), le transport de marchandises réalisé par les ménages lors de leurs achats motorisés. Sur cette même période (du lundi au vendredi), ils représentent de 50 à $55 \%$ des kilomètres générés par les marchandises et de 8 à $14 \%$ des flux totaux,

$\checkmark$ les flux de gestion urbaine (collecte des déchets, chantiers, services postaux...) représentent en moyenne de 10 à $15 \%$ des kilomètres générés par les marchandises et de 2 à $4 \%$ des flux totaux (en UVP).

Les déplacements d'achats des ménages étant décrits par les enquêtes ménages depuis les années 1970 et les flux de gestion urbaine se caractérisant par une grande volatilité nécessitant des enquêtes ciblées, les enquêtes " transport de marchandises en ville " se sont limitées au 


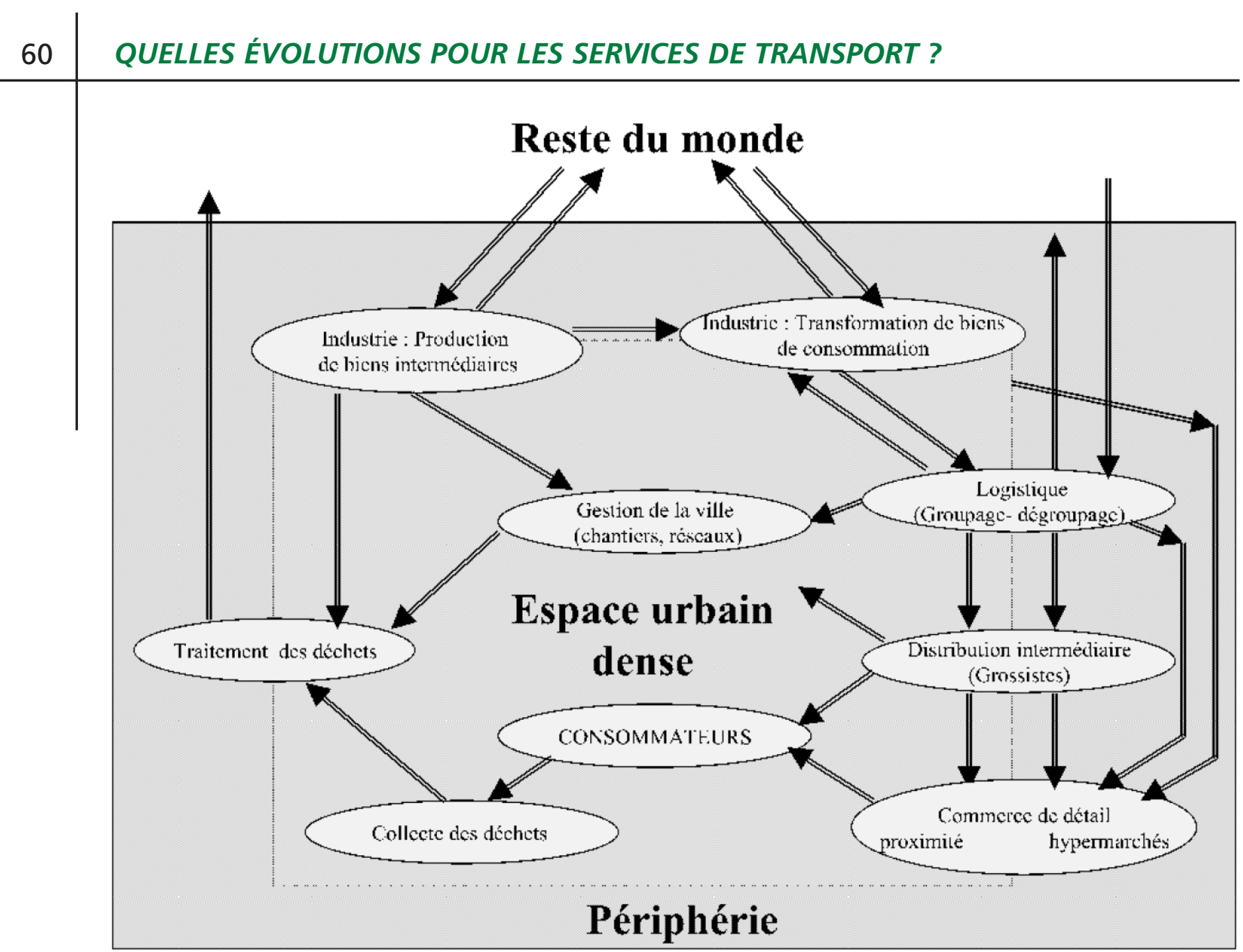

Schéma 1 : Les échanges de marchandises en ville : une multitude d'acteurs impliqués.

premier de ces trois segments : l'enlèvement et la distribution de marchandises dans les établissements industriels, commerciaux ou tertiaires des secteurs privé et public.

Le périmètre d'enquête idéal est celui de l'aire urbaine, qui rend compte du bassin d'activité et d'échanges de produits de la ville. Les contraintes financières et techniques font que ce périmètre se limite généralement à l'agglomération élargie aux communes voisines ayant un fort contingent de plates-formes logistiques ou à de gros établissements échangeant avec les communes de l'agglomération.

Le "mouvement ", au sens d'une expédition ou d'une livraison, ayant été choisi comme unité d'observation, il convient de pouvoir reconstituer les différentes occupations de la voirie qui peuvent en résulter, que ce soit à l'occasion du mouvement lui-même (caractéristiques et mode de stationnement du véhicule) ou pour décrire les déplacements entre deux mouvements. Le mouvement luimême est alors saisi auprès de l'établissement émetteur ou récepteur, les conditions d'approvisionnement le sont auprès des chauffeurs-livreurs et, plus généralement, les organisations logistiques le sont auprès des transporteurs. Les enquêtes se présentent ainsi sous la forme de trois enquêtes emboîtées les unes dans les autres, comme le résume le Schéma 2 de la page suivante.

L'enquête "Établissements " est administrée en premier. Chaque établissement (sélectionné dans l'aire d'étude selon une stratification fondée sur son activité et sa taille) est enquêté à l'aide de deux questionnaires selon une méthodologie proche des enquêtes " ménages " de déplacements, avec, d'une part, un questionnaire "Informations générales " qui recense toutes les caractéristiques de l'établissement et ses pratiques de livraisons ou d'expéditions et, d'autre part, un carnet de bord qui décrit, sur une semaine, l'ensemble des mouvements ayant concerné l'établissement. Cette enquête permet de repérer les variables de base de la génération des flux et, bien sûr, d'alimenter le modèle FRETURB.

Chaque livraison ou enlèvement consignés dans le carnet de bord donne lieu à la remise d'un questionnaire au chauffeur ayant desservi l'établissement. C'est l'enquête "Chauffeurs ", qui intervient donc en second lieu et qui permet de connaître le mode opératoire des personnes en charge du transport des marchandises (type de véhicule, nombre d'arrêts effectués sur le parcours, activités desservies, itinéraire...). 

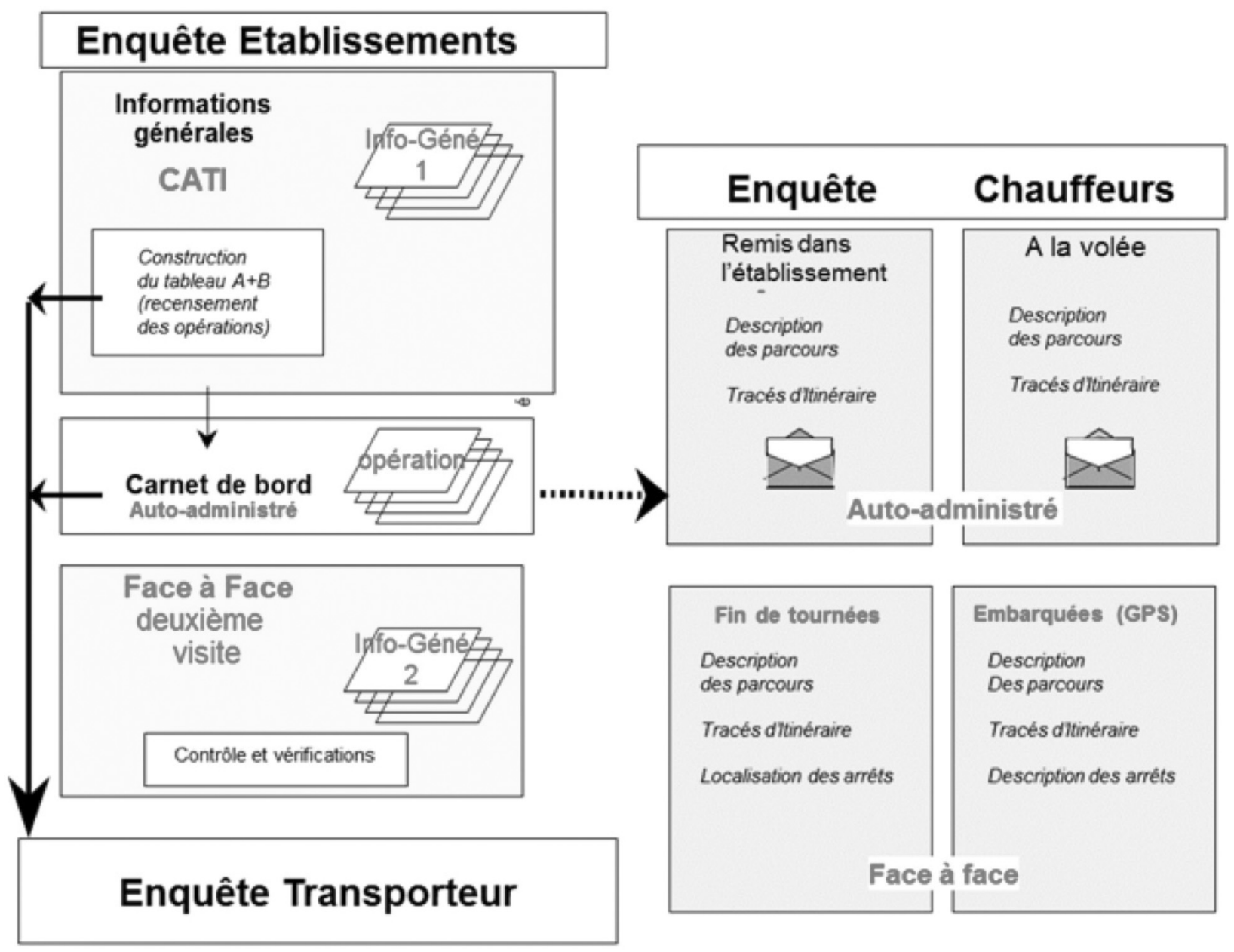

Schéma 2 : Les enquêtes emboîtées.

Cette enquête permet de repérer les entreprises de transport impliquées dans les livraisons urbaines. Elle sert ainsi à alimenter l'échantillon de l'enquête "Transporteurs ", dernier volet de l'enquête "Transports de marchandises en ville ". Cette dernière est indispensable pour comprendre la connexion entre les flux internes à la ville et les flux entrants et sortants en décrivant les principales organisations logistiques, en localisant les platesformes et en identifiant les rayons d'action dans l'ensemble de l'agglomération.

Les enquêtes "Chauffeurs " et "Transporteurs " vont également alimenter le modèle FRETURB et permettre, en particulier, de calculer la transformation des " mouvements " en occupation de la voirie, que ce soit pour les arrêts des véhicules ou pour leurs déplacements.

\section{Quelques résultats}

Cette première vague d'enquêtes réalisées à Marseille, à Bordeaux et à Dijon a apporté une masse inespérée de résultats inédits sur les transports de marchandises en ville. On peut en évoquer quelques-uns, qui portent sur trois aspects de cette activité, dont on aura compris qu'ils sont fortement articulés entre eux : la génération des mouvements, I'organisation logistique et le niveau d'occupation de la voirie.

En ce qui concerne la génération des mouvements, les trois villes sous enquête ont montré de fortes similarités, ce qui était très rassurant pour l'objectif de l'élaboration d'un modèle portable d'une ville à l'autre : le nombre des mouvements générés dans une agglomération est en effet directement lié à l'emploi, et ce quelle que soit la ville. On compte environ 1 mouvement par semaine et par emploi. Cette moyenne cache évidemment une très grande variabilité en fonction de l'activité : cela va des emplois de bureau, qui ne génèrent que 0,15 mouvement par semaine, jusqu'aux emplois dans les entrepôts, qui eux peuvent en générer plusieurs dizaines. En raison de leur importance dans l'emploi urbain, ce sont les commerces (de gros, de détail, ou les grandes surfaces) et le secteur tertiaire qui génèrent les deux tiers des mouvements de marchandises.

Sachant que les courbes représentent des dépendances statistiquement fortes, le graphique de la page suivante permet de comprendre que la modélisation des mouvements est très consistante dès lors qu'un fichier des établissements est disponible.

En ce qui concerne l'organisation logistique, on observe également de grandes similitudes entre les villes. C'est 


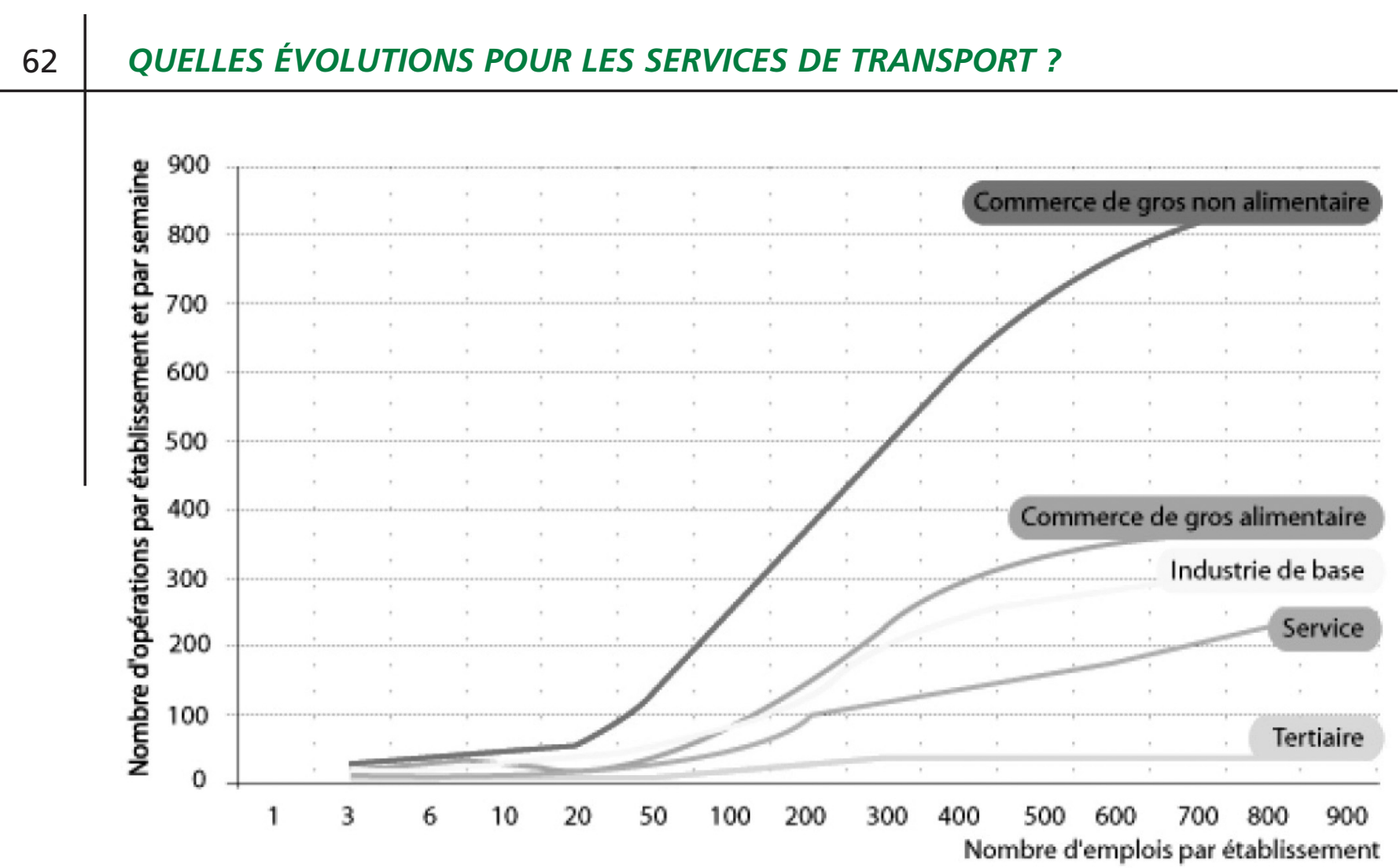

Graphique1 : La relation nombre de mouvements/nombre d'emplois selon l'activité de l'établissement.

ainsi que les tournées prédominent pour ce qui est des livraisons/enlèvements (75\%), mais elles représentent seulement un quart des parcours : cet écart est lié au fait que les tournées sont optimisées de manière à toucher un grand nombre d'établissements (en moyenne 13 par parcours, une valeur observée dans les trois villes sous enquête).

Réciproquement les traces directes représentent les trois quarts des parcours, pour seulement un quart des mouvements. Au sein de ces tournées, les activités desservies sont homogènes lorsqu'il s'agit de messagerie réguliè- re, ce qui n'est pas le cas des tournées de messagerie express, qui se caractérisent par des colis de petite taille.

On note également que le transport est majoritairement réalisé en compte propre, en particulier dans les plus grandes villes. Quant aux véhicules utilisés, ce sont des véhicules de moins de 3,5 tonnes dans plus de la moitié des cas.

Enfin, pour ce qui concerne l'occupation de la voirie et son impact sur le trafic, on note que les conditions de livraison sont homogènes dans les trois villes. Vues sous l'angle des horaires, elles sont partout calées sur les

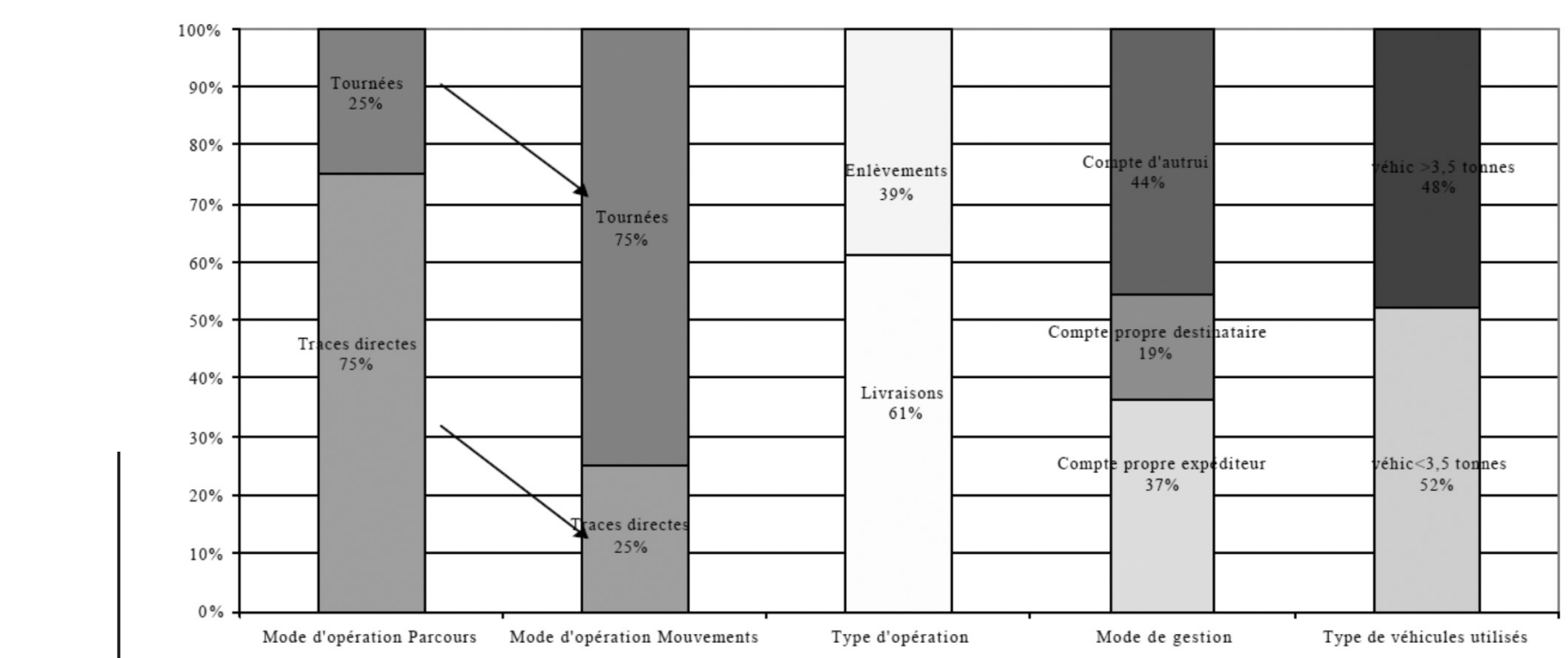

Graphique 2 : Les grandes caractéristiques des livraisons. 
heures d'ouverture des établissements, elles sont donc en décalé par rapport à celles des véhicules des particuliers (heure de pointe du matin de 10 à 11 heures, heure de pointe du soir de 16 à 17 heures).

La durée moyenne des arrêts est très proche d'une ville à l'autre, autour de 15 minutes, et, une fois sur deux, la manutention des marchandises se fait sans outil.

Notons que plus qu'à la taille de la ville, la durée de stationnement est sensible à l'étendue de la tournée : plus la tournée est longue, plus le temps de chargement/déchargement est court et devient similaire pour les trois villes. En centres urbains, cette durée d'occupation de la voirie par les véhicules à l'arrêt représente ainsi deux fois la durée d'occupation liée à la circulation des véhicules de livraison. Par conséquent, si, globalement, le transport de marchandises n'excède pas $10 \%$ du trafic automobile en ville, cette part peut atteindre $50 \%$ à certaines heures dans les zones les plus denses de l'agglomération. Circulation et stationnement se cumulent alors pour contribuer fortement à la consommation de l'espace viaire.

À travers ces quelques exemples de résultats, on perçoit mieux comment ces enquêtes ont permis de renseigner les principales fonctions caractéristiques du modèle FRETURB et comment ce dernier permet de simuler la formation des flux de transport de marchandises dans la ville de maniè- re suffisamment précise pour offrir, par exemple, aux aménageurs :

$\checkmark$ une image des lieux de génération des livraisons/enlèvements,

$\checkmark$ une évaluation des kilomètres parcourus par les véhicules de livraison en distinguant les véhicules utilitaires légers, les camions porteurs et les ensembles articulés,

$\checkmark$ la mesure de l'impact des véhicules à l'arrêt pour les livraisons, selon l'heure de la journée et les lieux fréquentés,

$\checkmark$ une identification de la part prise par les différents acteurs du système dans la formation des flux logistiques et de transport : activités desservies et celles pourvoyeuses de biens, opérateurs (selon leur mode de gestion et d'organisation du transport), activités riveraines impactées, etc.

Au-delà de ces restitutions de l'impact de la logistique urbaine sur l'occupation de la voirie (restitution qui, rappelons-le, ne nécessite plus d'enquête spécifique), le modèle FRETURB apporte également des possibilités de simulation qui sont largement utilisées par les aménageurs, qu'il s'agisse de jouer entre la réglementation locale, les espaces dévolus aux livraisons, les contraintes pesant sur les véhicules en centre-ville, la mise en œuvre de plates-formes mutualisées, etc.

\section{Durée moyenne d'un arrêt (mn)}

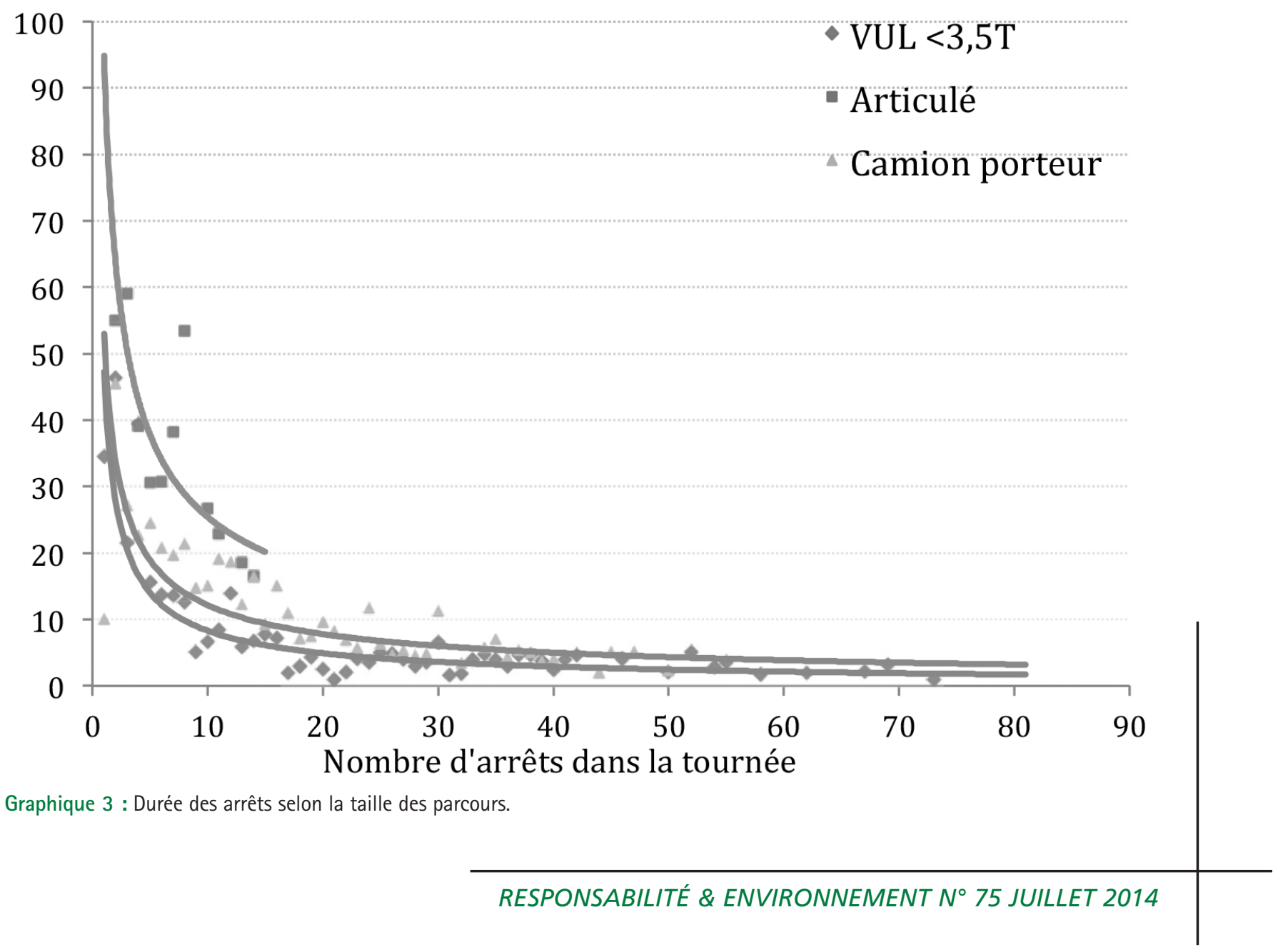




\section{QUELLES ÉVOLUTIONS POUR LES SERVICES DE TRANSPORT?}

Si l'on avait arrêté l'histoire de la connaissance statistique et de la modélisation des trafics urbains il y a deux décennies, on aurait pu résumer la situation en observant que la modélisation des transports de marchandises en ville avait pris un retard considérable par rapport à celle des voyageurs. La mise en œuvre sur le terrain des études que nous venons d'évoquer suggère que ce retard est en train de se réduire. La nouvelle vague d'enquêtes actuellement en cours devrait livrer ses premiers résultats dans quelques mois, qui permettront d'apprécier les invariants (vingt ans après la première vague !) ainsi que les adaptations des systèmes de logistique urbaine. Grâce à cette modélisation et à sa mise à jour statistique, il y a fort à parier que, demain, les marchandises transportées et livrées en ville ne seront plus le parent pauvre des politiques de transport urbain.

\section{Notes}

* Laboratoire d'Économie des Transports.

(1) De la société PTV (Allemagne).

(2) De la société Citilabs (États-Unis).

(3) De la société Caliper Corporation (États-Unis).
(4) De la société INRO (Canada).

(5) Laboratoire d'Économie des Transports (CNRS, ENTPE et Université Lyon 2).

(6) RUSSO (F.) \& COMI (A.), "A modeling system to simulate goods movements at an urban scale", Transportation, 37 (6), pp. 987-1009, 2010.

(7) ROUTHIER (J.L.) \& AUBERT (P.L.), FRETURB, un modèle de simulation des transports de marchandises en ville, $8^{\text {th }}$ WCTR Antwerp proceedings, vol. 1, pp. 531-544, Elsevier, 1999.

(8) Système Informatique pour le Répertoire des Entreprises et de leurs Établissements.

(9) Pour une présentation récente du modèle : ROUTHIER (J. L.) \& TOILIER (F.), "FRETURB : simuler la logistique urbaine ", in ANTONI (J. P.) (ed.), Modéliser la ville. Formes urbaines et politiques de transport, Paris, Economica, pp. 246-283, 2010.

Pour une présentation mathématique complète : BONNAFOUS (A.), GONZALEZ-FELIU (J.) \& ROUTHIER (J. L.), "An alternative UGM paradigm to OD matrices: the FRETURB model", in General Proceedings of the $13^{\text {th }}$ World Conference on Transport Research, 2013.

(10) De multiples rapports (ROUTHIER (J.-L.), AMBROSINI (C.) \& PATIER (D.)) ayant restitué ces résultats sont récapitulés dans l'ouvrage de Danièle PATIER, La logistique dans la ville, Paris, Éd. Celse, 2002. 